\title{
Réhabilitation respiratoire dans la bronchopneumopathie chronique obstructive
}

\author{
J.-L. Corhay, D. Dang Nguyen, T. Bury, F. Pirnay, R. Louis \\ Le traitement actuel de la bronchopneumopathie chronique obstructive (BPCO) doit comporter, outre un \\ traitement médicamenteux optimal, une réhabilitation respiratoire $(R R)$, de préférence multidisciplinaire, \\ et un programme de postrevalidation afin de maintenir les acquis. II est en effet clairement démontré \\ aujourd'hui que la RR permet d'améliorer la dyspnée, la tolérance à l'effort, l'activité physique et la \\ qualité de vie des patients. De même, elle réduit le recours aux soins de santé et donc le coût de la \\ maladie. Dans cette synthèse, nous présentons ce qu'est la réhabilitation pulmonaire, ses indications et \\ ses résultats, et la façon dont elle se déroule en ambulatoire.
}

○ 2011 Elsevier Masson SAS. Tous droits réservés.

Mots clés : BPCO ; Réhabilitation respiratoire ; Traitement de la BPCO

Plan

\section{- Introduction}

- Définition

- Dysfonctionnement musculaire squelettique

dans la bronchopneumopathie chronique obstructive

Changements morphologiques et structuraux

- Indications de la réhabilitation respiratoire

dans la bronchopneumopathie chronique obstructive

- Contre-indications à la réhabilitation respiratoire

dans la bronchopneumopathie chronique obstructive

- Personnels et équipements nécessaires

Intervenants dans le programme de réhabilitation respiratoire

Équipement nécessaire minimal

- Modalités de la réhabilitation respiratoire

- Composantes d'un programme de réhabilitation respiratoire Réentraînement à l'effort

Renforcement des muscles spécifiques

Gymnastique et assouplissement

Éducation du patient

Kinésithérapie respiratoire

Mesures diététiques et autres interventions

- Efficacité de la réhabilitation respiratoire

Effets sur la qualité de vie

Effets sur la dyspnée

Effets sur la tolérance à l'effort

Effets sur l'activité physique

Effets sur les muscles respiratoires

Effets sur les muscles des membres supérieurs

Effets sur la dépression et l'anxiété

Effets sur la consommation médicale et les exacerbations

Effets sur la fonction respiratoire et déclin annuel du VEMS

Effets sur le poids

Effets sur la survie

- Conclusion

\section{Introduction}

La bronchopneumopathie chronique obstructive (BPCO) est définie comme une maladie respiratoire chronique que l'on peut prévenir et traiter associée à quelques effets systémiques significatifs qui contribuent à sa sévérité. Sa composante pulmonaire est caractérisée par une limitation des débits aériens qui n'est pas totalement réversible. L'obstruction, généralement progressive, est associée à une réaction inflammatoire anormale des poumons suite à l'inhalation de particules et de gaz toxiques [1]. Bien que la BPCO touche les poumons, cette affection a également des conséquences systémiques significatives [2]. Parmi ces dernières, on relève la diminution de la capacité à l'effort, l'atrophie musculaire, l'ostéoporose, la perte de poids, les maladies cardiovasculaires et la dépression. La prise en charge thérapeutique du patient doit tenir compte à la fois de la composante pulmonaire de la BPCO, mais aussi de la composante systémique ${ }^{[1]}$.

La BPCO représente un lourd fardeau pour la santé publique et le patient qui en souffre. D'évolution progressive, elle entraîne un cercle vicieux (Fig. 1) ${ }^{[3]}$ où l'inactivité physique, la crainte et l'isolement social aggravent le handicap respiratoire et le déconditionnement. Ce déconditionnement dans la BPCO s'observe dès les premiers stades de la maladie et constitue un des effets systémiques de la maladie. L'intolérance à l'effort dont se plaint la majorité des patients BPCO peut s'expliquer en grande partie par des facteurs limitants non seulement respiratoires, mais aussi périphériques. Ainsi, l'atteinte des muscles squelettiques périphériques ${ }^{[4]}$ joue un rôle primordial dans cette limitation à l'effort des patients BPCO.

Dans la prise en charge thérapeutique de la BPCO, la réhabilitation respiratoire (RR) a aujourd'hui acquis ses lettres de noblesse et en est devenue une des composantes essentielles. Elle est proposée dans tous les consensus internationaux sur la BPCO, au même titre que les traitements médicamenteux. Elle permet en effet de rompre le cercle vicieux décrit ci-dessus (Fig. 1). La RR est un programme pluridisciplinaire qui prend en compte l'ensemble des répercussions de la pathologie dans un programme adapté à chaque patient. Elle reste néanmoins sousutilisée par le corps médical, en raison d'une part d'une méconnaissance de la RR et de ses indications [5], et d'autre part en raison d'une indifférence des médecins pour les traitements 


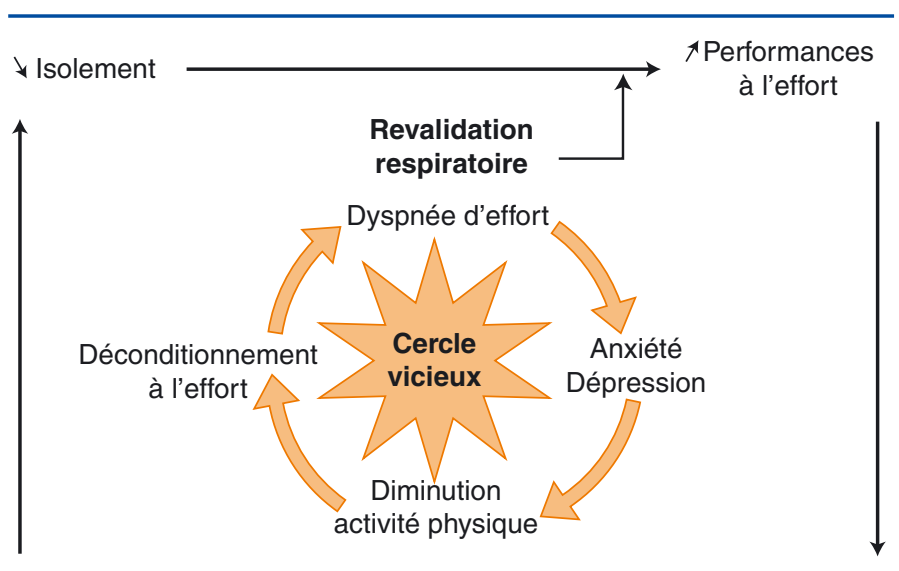

$₫$ Confiance en soi

$\downarrow$ Dyspnée d'effort

EMC

Figure 1. Spirale du déconditionnement à l'effort dans la bronchopneumopathie chronique obstructive et effet de la réhabilitation respiratoire (reproduit avec l'aimable autorisation de l'éditeur ${ }^{[3]}$ ).

non médicamenteux. Un des buts de cette revue est donc d'aider à modifier la perception des médecins à l'égard de la RR.

\section{Définition}

La définition de la RR donnée par l'American Thoracic Society et l'European Respiratory Society (ATS/ERS [2006]) est la suivante : "La réhabilitation respiratoire est un ensemble de soins personnalisés, basés sur des évidences scientifiques, dispensés par une équipe multidisciplinaire à des patients atteints d'une maladie respiratoire chronique, qui sont symptomatiques et ont souvent des activités de la vie quotidienne diminuées. Intégrée dans le traitement individualisé du patient, la RR a comme objectif de réduire les symptômes respiratoires, optimiser le statut fonctionnel, améliorer l'intégration sociale et réduire les coûts de santé en stabilisant ou en réduisant les manifestations systémiques de la maladie » ${ }^{[6]}$.

La RR s'inscrit donc dans une approche multidisciplinaire incluant bien évidemment l'entraînement physique à l'effort et le renforcement des muscles périphériques qui en constituent des éléments essentiels, l'ergothérapie, l'éducation du patient, le sevrage tabagique, le conseil diététique et l'accompagnement psychosocial. Il s'agit donc d'une vraie prise en charge globale du patient BPCO. Si une partie de la RR peut être réalisée en cours d'hospitalisation ou même au domicile, c'est en ambulatoire que les programmes de RR sont les plus efficaces.

\section{Dysfonctionnement musculaire squelettique dans \\ la bronchopneumopathie chronique obstructive}

Parmi les complications systémiques de la BPCO, la dysfonction musculaire périphérique a une place très importante; elle est caractérisée par l'atrophie des muscles, une diminution de la force musculaire et une baisse de la capacité oxydative. Ces changements musculaires sont associés à une tolérance à l'effort réduite, une dégradation de la qualité de vie et à une réduction d'espérance de vie et ce, de façon indépendante à l'insuffisance de la fonction pulmonaire [7]. Au début des années 1990, Jones et al. ont en effet montré que, pour une fonction pulmonaire donnée, il existe une grande variation dans la capacité maximale à l'exercice ${ }^{[8]}$. Plus tard, Killian et al. ont rapporté qu'une proportion significative de patients BPCO présentait comme cause essentielle de l'arrêt de l'effort, une fatigue ressentie au niveau des membres inférieurs plutôt qu'une sensation d'essoufflement ${ }^{[9]}$. Enfin, des recherches plus récentes se sont attachées à prédire les changements morphologiques et structuraux qui surviennent au niveau des muscles squelettiques périphériques du patient BPCO. Quant à l'origine de l'atteinte musculaire périphérique, elle paraît multifactorielle et l'importance relative des différents facteurs varie probablement d'un patient à l'autre. Ainsi, les causes les plus probables de cette dysfonction dans la BPCO sont: le déconditionnement, l'inflammation systémique, l'hypoxémie, la dénutrition, le stress oxydatif et la prise médicamenteuse (surtout la corticothérapie systémique).

\section{Changements morphologiques et structuraux}

Au contraire de l'atteinte pulmonaire qui est en grande partie irréversible, l'atteinte musculaire périphérique représente une cause potentiellement traitable de l'intolérance à l'effort rencontrée chez les patients BPCO. Sur le plan morphologique, les principaux changements observés concernent la diminution du volume des fibres musculaires, une modification de l'organisation métabolique des fibres musculaires (au détriment des fibres oxydatives) ainsi qu'une diminution de la capillarisation de ces fibres [10, 11]. La prévalence de l'atrophie musculaire périphérique chez le patient BPCO varie de $20 \%$ à $40 \%$ selon les études et est proportionnelle à la sévérité de la maladie respiratoire. Elle serait plus fréquemment présente chez la femme que chez l'homme [12]. La typologie musculaire a été évaluée chez les patients BPCO par les techniques classiques de typage histochimique. Dans le décours de la maladie respiratoire, dans un premier temps, la proportion de fibres de type I demeure inchangée, puis on constate progressivement une diminution de la proportion de fibres de type I et de type IIa, associée à une augmentation des fibres de type IIb [13]. Tous les muscles ne sont cependant pas affectés de la même façon. Généralement, le déclin de la force musculaire des membres supérieurs est moindre, comparé à celui des membres inférieurs. Quant à l'adaptation du diaphragme chez les sujets BPCO, elle diffère de celle observée au niveau des muscles périphériques. En effet, alors que les muscles périphériques atteints présentent une capacité de métabolisme aérobie faible et une moins grande tolérance à la fatigue, les modifications structurelles observées dans le diaphragme se caractérisent par une proportion accrue de fibres résistantes à la fatigue [14]. Sur le plan métabolique, l'efficacité du métabolisme oxydatif au niveau des muscles périphériques est réduite suite à une diminution de l'activité de la plupart des enzymes oxydatives mitochondriales (par exemple, la succinate déshydrogénase ou la citrate synthase). Le nombre et la taille des mitochondries dans les muscles squelettiques seraient également réduits ${ }^{[15]}$. Ce sont bien sûr ces différents changements morphologiques et fonctionnels qui expliquent les modifications du métabolisme musculaire que l'on observe durant l'exercice chez le patient souffrant de BPCO. Il existe donc chez ce patient un déficit de production d'adénosine triphosphate (ATP) par le processus de phosphorylation oxydative alors que l'on décrit une activation précoce de la glycolyse anaérobie.

Il existe deux conséquences fonctionnelles majeures secondaires à ces différents changements morphologiques et structuraux :

- réduction de la filière aérobie (endurance) au sein des muscles périphériques, expliquant en partie l'aggravation de la dyspnée du patient BPCO ;

- atrophie musculaire induisant une diminution de la force musculaire, en particulier au niveau des membres inférieurs.

\section{Indications de la réhabilitation respiratoire dans la bronchopneumopathie chronique obstructive}

La RR s'adresse à tous les patients souffrant d'une maladie respiratoire chronique quelle qu'en soit l'origine et qui ont une atteinte de la fonction respiratoire entraînant une dyspnée et une intolérance à l'effort malgré un traitement pharmacologique optimal. 
66

\section{Point fort}

Maladies pouvant bénéficier d'une RR

- BPCO

- Asthme sévère

- Mucoviscidose

- Pneumopathies interstitielles fibrosantes

- Cancer bronchique opéré

- Maladies neuromusculaires avec restriction

- Avant et après transplantation pulmonaire ou chirurgie

de réduction de l'emphysème

Même les candidats à une chirurgie de réduction de l'emphysème ou à la transplantation pulmonaire sont de très bons candidats. La RR peut être proposée en état stable ou après une exacerbation. Les buts de la RR sont de soulager les symptômes, de restaurer au mieux les capacités physiques et l'autonomie, de diminuer le handicap et par conséquent d'améliorer la qualité de vie des patients. En ce qui concerne la BPCO, le Global Initiative for Chronic Obstructive Lung Disease (GOLD) la recommande dès le stade 2 (volume expiratoire maximal/ seconde [VEMS] $<80 \%$ des valeurs prédites et un rapport VEMS/capacité vitale $[\mathrm{CV}]<70 \%$ ), c'est-à-dire même en l'absence de symptômes dès lors que la fonction respiratoire est perturbée. Cependant, en accord avec la définition conjointe de l'ATS/ERS de 2006, tous les patients souffrant des conséquences systémiques de la BPCO sont de bons candidats pour la RR [6]. Le remboursement de la $\mathrm{RR}$, dans ces différentes modalités, dépend bien sûr des moyens financiers mis à disposition et des règles érigées par la Sécurité sociale de chaque pays.

\section{6 \\ Point fort}

Les candidats optimaux à la RR sont les patients motivés dont la tolérance à l'effort est limitée, la qualité de vie réduite, restant dyspnéiques et ayant une consommation médicale importante.

\section{Contre-indications}

\section{à la réhabilitation respiratoire dans la bronchopneumopathie chronique obstructive}

Les contre-indications principales sont le manque de motivation, les troubles psychiatriques ou cognitifs sévères, une comorbidité instable (cardiopathie, insuffisance hépatique, etc.), une incapacité à réaliser l'exercice (polyarthrose sévère par exemple). Si aux États-Unis certains assureurs refusent le remboursement de la RR en cas de tabagisme actif [16], il ne s'agit pas pour nous d'une contre-indication. Il est par ailleurs prouvé que les patients qui continuent de fumer retirent les mêmes bénéfices de la RR; ils sont néanmoins invités à entreprendre un sevrage tabagique. Cependant, l'adhérence à la RR des fumeurs reste généralement moindre comparée à celle des ex-fumeurs [17]. L'âge ${ }^{[18,19]}$ et l'importance du degré de l'obstruction bronchique [20] ne constituent pas des contreindications à la RR. L'hypoxémie sévère à l'effort doit être préalablement corrigée par une oxygénothérapie ; la ventilation non invasive continue ou intermittente ne constitue pas non plus une contre-indication au suivi d'un programme de RR. Peu d'études ont établi des facteurs prédictifs de mauvaise adhérence. On retient cependant, en plus du tabagisme actif, $l^{\prime}$ isolement social [21]. Le taux d'abandon de la RR rapporté par la majorité des études est de l'ordre de $20 \%$ et les raisons citées sont souvent des affections intercurrentes (exacerbations sévères de BPCO, hospitalisations, etc.) et des problèmes de logistiques (transport, coût pour le malade, éloignement du centre, etc.). En cas d'absence de transport adéquat, il faut envisager une RR durant une hospitalisation.

\section{Personnels et équipements nécessaires}

\section{Intervenants dans le programme de réhabilitation respiratoire}

La RR est une prise en charge globale des patients; elle nécessite donc une équipe multidisciplinaire plus ou moins étoffée de professionnels de la santé, coordonnée par le médecin et centrée sur le patient. En termes de nombre d'intervenants paramédicaux, on a suggéré un intervenant pour 4 à 8 patients pour le réentraînement à l'effort et un intervenant pour 8 à 16 patients pour l'éducation thérapeutique [6]. Ces différents acteurs se réunissent régulièrement pour discuter du cas du patient, de ses progrès éventuels, afin d'optimiser son traitement. Même si les programmes de RR n'engendrent que rarement des complications sérieuses, tous les intervenants paramédicaux doivent être rompus aux techniques de réanimation cardiorespiratoires. Nous présentons les différents acteurs impliqués dans ce programme.

\section{6 Point fort}

La RR doit être réalisée par une équipe multidisciplinaire.

\section{Médecin}

Le service est de préférence dirigé par un pneumologue agréé en RR aidé d'un ou de plusieurs collaborateurs. Il assure le suivi médical et le contact avec les médecins extérieurs au centre. Il prescrit le bilan initial, pose l'indication et les modalités de la $\mathrm{RR}$, introduit les dossiers auprès de l'organisme de remboursement, recherche les comorbidités qui pourraient interférer avec le bon déroulement de la RR et participe à l'éducation du patient. Au besoin, il optimalise le traitement médical du patient admis dans un programme de RR.

\section{Kinésithérapeutes}

Le kinésithérapeute assiste le médecin pour la réalisation des tests d'évaluation au début et en fin du programme de RR. Il surveille constamment le comportement général, cardiaque et respiratoire du patient au cours des efforts. Il s'occupe également des séances de gymnastique, de relaxation, des techniques respiratoires (lèvres pincées, respiration diaphragmatique) et de drainages bronchiques ou des techniques de lutte contre l'hyperventilation.

\section{Ergothérapeute}

La démarche de l'ergothérapeute repose tout d'abord sur un bilan d'autonomie du patient afin de permettre un programme personnalisé. L'ergothérapeute peut ensuite améliorer la vie de tous les jours du patient d'une part en lui donnant des conseils et des petits "trucs» l'aidant à mieux gérer son souffle, et d'autre part en lui présentant des moyens d'aides techniques ou en le guidant dans l'achat de certains matériels (chausse-pied à long manche, utilisation d'un déambulateur, pince ramasseobjet, etc.). Ainsi, un programme d'aide à la marche au travers de dispositifs de soutien (par exemple le rollator) peut aider un patient qui aurait des difficultés à se déplacer [22, 23] (Fig. 2). L'ergothérapeute joue aussi un rôle essentiel dans l'éducation du patient oxygénodépendant. 


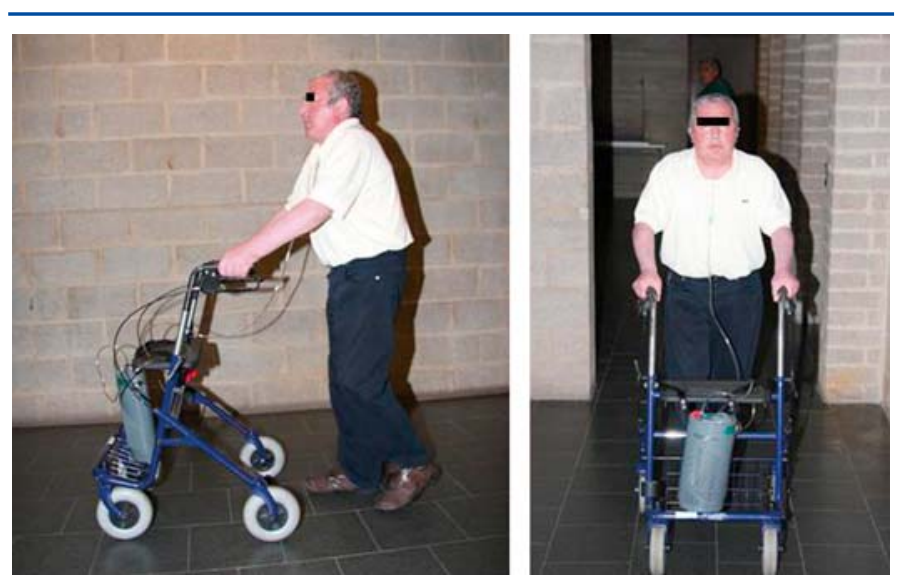

Figure 2. Patient atteint de bronchopneumopathie chronique obstructive en cours de réhabilitation respiratoire aidé d'un déambulateur et d'administration d'oxygène à l'aide d'un portable d'oxygène liquide.

\section{Nutritionniste}

La dénutrition est fréquente dans la BPCO ; elle s'accompagne d'un état hypercatabolique qui peut même conduire à la cachexie. De $30 \%$ à $40 \%$ des patients référés dans un centre de RR ambulatoire ont une diminution de leur masse corporelle évaluée par une mesure de l'indice de masse corporelle (IMC: rapport du poids sur la taille en mètres au carré). La masse corporelle se compose principalement de la masse grasse et de la masse maigre (os, viscères, muscles). La masse dite "maigre " est un bon reflet de la masse musculaire et peut facilement être estimée par impédancemétrie. La diminution de cette dernière et de l'IMC sont un facteur de risque indépendant de mortalité dans la BPCO [24, 25], de limitation à l'effort [26] et de diminution de la qualité de vie ${ }^{[27]}$. En réalisant une anamnèse alimentaire fouillée et en déterminant la masse grasse et maigre par impédancemétrie en début de RR, le nutritionniste peut conseiller le patient afin de pallier aux excès alimentaires, mais surtout pallier aux carences. Une étude récente a montré le bénéfice de la combinaison de conseils diététiques et de compléments nutritifs, en dehors d'un programme de RR, chez des patients BPCO en sous-poids ${ }^{[28]}$.

\section{Psychologue}

La majorité des patients en RR présentent des troubles anxiodépressifs francs. Les raisons ne manquent pas : mauvais état de santé, hospitalisations ou interventions médicales fréquentes, isolement social, perte de perspectives, difficultés à réaliser des gestes de la vie quotidienne, frustrations, etc. Un questionnaire tel que l'Hospital Anxiety and Depression Scale (HADS) peut permettre de reconnaître et de quantifier des signes d'anxiété et de dépression [29]. L'évaluation de ces différentes composantes et par là l'optimalisation de leur prise en charge par l'ensemble de l'équipe est réalisée par le psychologue. Occasionnellement, en cas de syndrome anxiodépressif sévère, les patients peuvent être orientés vers un psychiatre qui se charge de réaliser une psychothérapie ou introduit un traitement médicamenteux adéquat. Indissociable de la prise en charge multidisciplinaire, l'intervention du psychologue est recommandée par les comités d'experts et ne serait pas efficace en single therapy [30]. De plus, le psychologue participe encore à la lutte contre diverses assuétudes (tabagisme et alcoolisme).

\section{Assistant social}

La maladie dont souffrent les patients entrant dans un programme de RR entraîne souvent de lourdes charges financières et administratives. L'assistant social peut aider les patients dans leurs différentes démarches en vue d'obtenir les interventions légales auxquelles ils ont droit. L'assistant social recherche aussi, pour ces patients à autonomie réduite, une aide aux déplacements vers le centre de réhabilitation.

\section{Infirmière}

Le personnel infirmier spécialisé dans les soins respiratoires peut jouer un rôle important essentiellement chez les patients hospitalisés, non seulement pour l'administration des soins, mais aussi pour l'éducation et la surveillance du réentraînement à l'effort.

\section{Équipement nécessaire minimal [31]}

Le site doit se trouver dans un environnement approprié, avoir des critères d'espace et de confort suffisants (vestiaires, douches, salles d'examen et de réentraînement, etc.), ainsi qu'un accès facile pour les personnes handicapées (rampe d'accès, parking réservé, etc.).

L'équipement doit comporter au minimum :

- des ergocycles et des tapis roulants ;

- des tensiomètres, des cardiofréquencemètres et des oxymètres de pouls ;

- un électrocardiographe 12 dérivations;

- des sources d'oxygène (oxygène mural, portables d'oxygène liquide, cylindres);

- du matériel de réanimation ;

- un local de soins d'urgence ;

- un accès à la spirométrie, à l'analyse des gaz du sang artériel ;

- des supports d'éducation (posters, télévision, lecteur DVD, projecteur de diapositives, etc.) ;

- des questionnaires d'évaluation de la dyspnée (échelle analogique visuelle, échelle de Borg) et de la qualité de vie (génériques ou spécifiques) ;

- du petit matériel de gymnastique (haltères, bâtons, élastiques, etc.).

S'ajoute à cela :

- un couloir de 30 mètres pour la réalisation du test de marche de 6 minutes ;

- un accès à la radiographie thoracique, au matériel d'épreuve d'effort cardiorespiratoire ;

- un analyseur de CO ;

- des appareils permettant la réalisation d'électrostimulation et du renforcement musculaire segmentaire.

Nous insistons sur la nécessité de disposer d'un chariot de réanimation et d'un accès au Service mobile d'urgence et de réanimation (Smur) permettant de transférer au besoin les patients vers un service d'urgence ou de réanimation.

\section{Modalités de la réhabilitation respiratoire}

La RR peut être réalisée lors d'une hospitalisation, en ambulatoire dans un centre rattaché à une institution hospitalière, voire même au domicile du patient ${ }^{[6,32]}$. Dans cette dernière situation, le patient ne bénéficie pas de la dynamique de groupe et de la même sécurité que dans les deux autres modalités; de plus, la RR reste alors limitée au réentraînement à l'exercice. Cependant, une étude récente de Maltais a relancé l'intérêt d'une RR au domicile supervisée, comme alternative équivalente à la RR en ambulatoire à l'hôpital [33]. Finalement, le choix dépend de l'état du patient, de sa motivation, des disponibilités locales ou des possibilités financières. En ambulatoire, la durée du programme doit être au minimum de 6 à 8 semaines à raison de trois séances par semaine pour obtenir un bénéfice. En revanche, la RR en hospitalisation s'effectue sur une durée plus courte, en général de 2 à 3 semaines, surtout réservée aux patients ne pouvant se déplacer et ayant un déconditionnement sévère ; elle reste de loin plus coûteuse pour la société que la RR menée en ambulatoire. Le GOLD recommande un programme d'au moins 6 semaines et considère que l'efficacité de la RR est d'autant plus grande que sa durée est importante [1]. Dans notre centre, nous préférons la RR ambulatoire et sur une durée allant jusqu'à 6 mois. De plus, nous constatons que chez certains de nos patients, la «rupture » par rapport au milieu sociofamilial et à ses habitudes peut être bénéfique. Rappelons encore que la majorité des études ayant démontré les bénéfices de la RR a été menée lors de programmes ambulatoires. 
Nous décrivons ici succinctement le déroulement de la RR telle qu'elle se pratique dans notre centre. Après quelques tests d'entrée (épreuves fonctionnelles respiratoires complètes, test de marche de 6 minutes, épreuve triangulaire de type maximal par ergospirométrie, mesure de la puissance des muscles inspiratoires et expiratoires, mesure de la force du quadriceps, mesure de la masse maigre par impédancemétrie, questionnaires sur la qualité de vie), la RR démarre pour une durée de 6 mois (60 séances de 2 à 3 heures, trois fois par semaine les 3 premiers mois, puis à raison de deux fois par semaine les mois suivants). Les efforts sont adaptés à chaque patient et selon leur capacité estimée par les tests préalables. Les membres de l'équipe surveillent constamment le comportement général des patients (fréquence cardiaque, saturation en $\mathrm{O}_{2}$, pression artérielle, fréquence respiratoire, mode de ventilation). Le but est un entraînement progressif et régulier en évitant les efforts excessifs. En concertation multidisciplinaire est dressé périodiquement pour chaque patient un tableau de ses progrès afin d'adapter au mieux le programme de RR. En fonction des résultats, nous avons la possibilité, après réévaluation, de reprendre la RR après 1 an.

\section{- Composantes d'un programme de réhabilitation respiratoire}

\section{Réentraînement à l'effort}

Il s'agit de la pierre angulaire de la prise en charge en RR et elle consiste en un entraînement d'endurance en aérobie réalisé par le biais du tapis roulant de marche ou de la bicyclette ergométrique.

Cet entraînement d'une durée de 40 à 60 minutes peut être réalisé en continu à une haute intensité située entre $50 \%$ et $80 \%$ de la fréquence cardiaque maximale ou de la puissance maximale obtenue lors de l'épreuve cardiorespiratoire maximale de départ. Un exercice de haute intensité est en effet nécessaire pour rendre réversibles les anomalies des muscles squelettiques par une activation du stimulus anabolique. Tous les patients ne sont cependant pas en état d'effectuer de tels exercices. La séquence de temps peut alors être fragmentée en quatre périodes de 10 à 15 minutes. On peut aussi, chez la plupart des patients, proposer un entraînement par intervalle : l'exercice est alors effectué avec une intensité élevée ( $>80 \%$ de la puissance maximale, extrêmes : $80 \%$-150 \%) sur des séquences de temps beaucoup plus courtes ( $20 \mathrm{~s}$ à $3 \mathrm{~min}$ ) suivies d'une période de temps souvent similaire à une intensité plus faible (30 \% à $75 \%$ de la puissance maximale) (Fig. 3), ces alternances étant répétées plusieurs fois ${ }^{[34]}$. Le réentraînement à l'effort dit "en intervalle » est parfois mieux toléré [35] et permet d'obtenir au minimum les mêmes résultats que ceux obtenus avec l'entrâ̂nement en continu $[34,36]$. De plus, il permettrait également d'obtenir une meilleure adhérence à long terme du patient à son programme de réentraînement [35]. Comme dans tout programme d'entraînement, la charge de travail imposée au patient doit être adaptée au fur et à mesure des progrès réalisés par le patient. En pratique clinique, nous utilisons les scores de symptômes pour ajuster le niveau d'effort : un niveau allant de 4 à 6 sur l'échelle de Borg pour la fatigue ou la dyspnée semble être un objectif raisonnable [37].

\section{Renforcement des muscles spécifiques}

\section{Membres inférieurs}

Le pédalage et la marche sont souvent insuffisants pour renforcer les quadriceps. Des exercices spécifiques sont dès lors proposés afin de les renforcer : travail à des charges faibles sur des bancs de musculation (deux à quatre séries de six à 12 répétitions à une intensité de $50 \%$ à $80 \%$ de la charge maximale supportée par le patient lors d'une seule élévation). Ces exercices en résistance n'engendrent en général que très peu de dyspnée par rapport à l'entraînement aérobie et sont donc très bien tolérés par les patients.
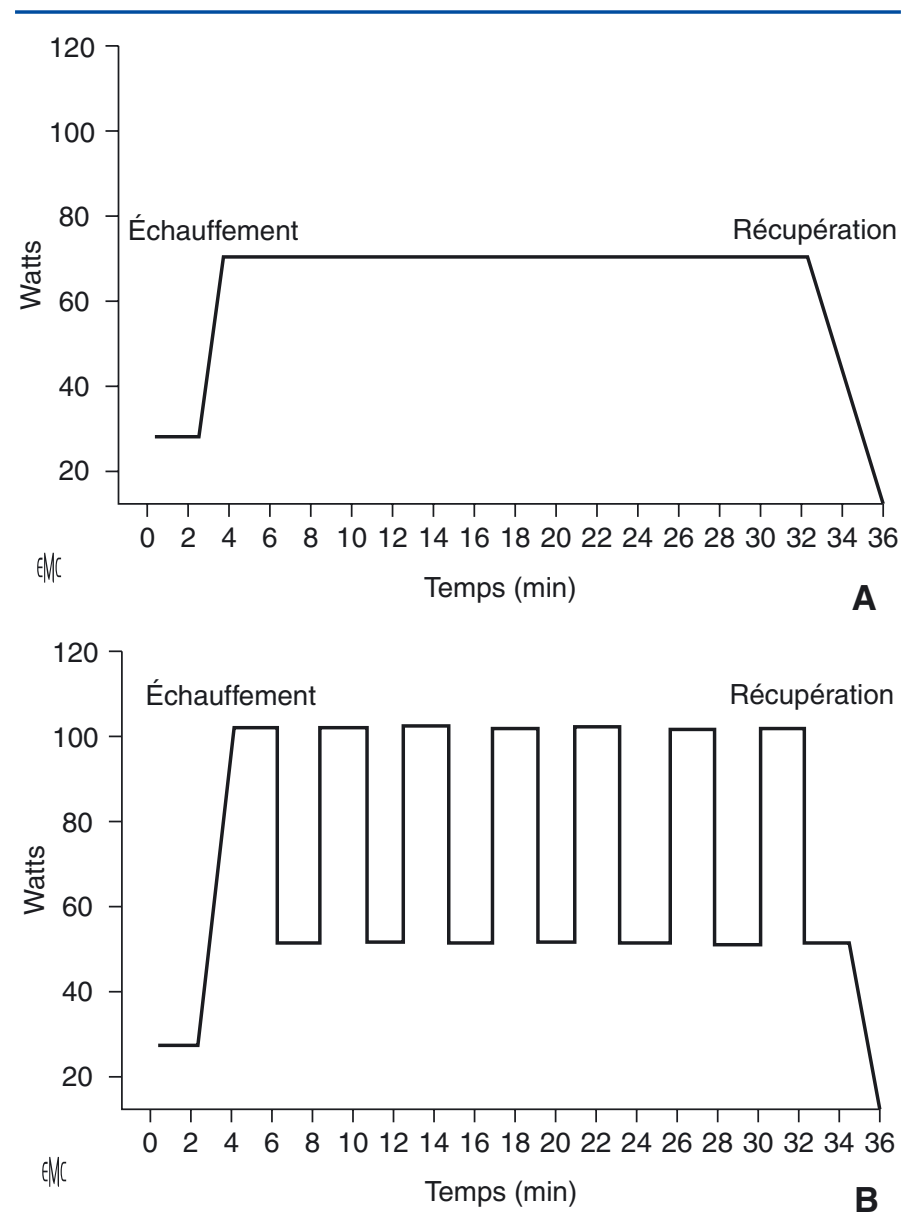

Figure 3. Exemples de protocoles de réentraînement à l'effort. A. Exercice continu à $70 \%$ de la capacité maximale à l'effort.

B. Exercice en créneaux de 2 minutes d'une intensité maximale puis de 2 minutes d'une intensité plus faible.

\section{Électrostimulation neuromusculaire (ESNM)}

Les patients BPCO sont cependant souvent trop faibles et dyspnéiques pour tolérer une intensité de travail musculaire entraînant des gains. Or, pendant un renforcement musculaire par ESNM, la sollicitation cardiopulmonaire est plus basse que lors d'un entraînement classique en résistance [38]. Ainsi, l'ESNM (deux à trois séances par semaine durant 4 à 6 semaines) engendre une sensation de dyspnée et de fatigue musculaire plus acceptable, tout en améliorant la force musculaire et la tolérance à l'effort, chez des patients BPCO sévèrement déconditionnés et dénutris ${ }^{[39]}$.

\section{Renforcement de muscles des membres supérieurs}

Il est systématiquement réalisé (haltères, élastiques et ergomètre à bras) car beaucoup d'activités de la vie courante sollicitent les membres supérieurs.

\section{Muscles inspiratoires}

Même si les études sont encore trop peu nombreuses sur ce sujet, il est recommandé de proposer aux patients présentant une diminution de la force des muscles inspiratoires un entraînement spécifique des muscles inspiratoires [30]. Plusieurs types de réentraînement ont été proposés mais aucun d'eux n'est à privilégier plus qu'un autre : hyperpnée isocapnique, respiration contre une résistance (dispositif simple consistant à placer sur le circuit inspiratoire un orifice de petit diamètre) ou valve inspiratoire à seuil de déclenchement réglable ne s'ouvrant que pour un niveau prédéterminé de pression (Threshold ${ }^{\circledR}$ IMT). Les avantages majeurs de l'entraînement des muscles inspiratoires sont qu'il s'agit d'une technique simple et peu coûteuse, utilisable chez les patients n'ayant pas accès à une structure de $\mathrm{RR}$, ou en complément de celle-ci. 


\section{Gymnastique et assouplissement}

Elle est le plus souvent réalisée de manière collective. C'est un complément incontournable, car d'autres facettes de la capacité fonctionnelle méritent d'être réhabilitées : souplesse articulaire, équilibre, proprioception et coordination.

\section{Éducation du patient}

Les séances d'éducation permettent au patient d'acquérir des connaissances qui vont l'aider à prendre en charge sa maladie. Ainsi, la sensibilisation et la responsabilisation du patient dans sa prise en charge thérapeutique entraînent une diminution du nombre de réadmissions à l'hôpital [40,41]. Les sujets abordés sont très divers : traitement de la BPCO, oxygénothérapie, techniques d'économie de l'énergie (ergothérapeute), techniques de respiration et de relaxation, nutrition, voyages, gestion du stress, sexualité, conduite à tenir en cas d'urgence, fin de vie.

\section{Kinésithérapie respiratoire}

En cas d'encombrement bronchique, elle permet de favoriser l'élimination des sécrétions bronchiques en excès qui ne font qu'augmenter l'inconfort du patient. De plus, les kinésithérapeutes réalisent systématiquement une évaluation permettant une éventuelle modification du mode ventilatoire (par exemple : expiration avec lèvres pincées).

\section{Mesures diététiques et autres interventions}

Il faut associer les suppléments nutritifs et conseils diététiques au réentraînement à l'effort si l'on veut améliorer la masse maigre des patients BPCO. Ainsi, suite à un suivi diététique adapté, certains patients BPCO dénutris augmentent leur poids corporel et améliorent donc leur pronostic vital [27]. Le recours à un psychologue, voire à un psychiatre, est nécessaire en cas de dépression, d'angoisse et d'anxiété en vue d'un traitement adapté. Le soutien psychosocial permet aussi d'aider le patient à accepter sa maladie et à améliorer son adhérence à l'ensemble du traitement, y compris la RR [17]. Une aide au sevrage tabagique et des séances d'ergothérapie peuvent aussi faire partie du programme.

66

$$
\text { Point fort }
$$

\section{Recommandations de I'ATS/ERS 2006 pour améliorer les performances à I'effort lors d'un programme de $\mathbf{R} \mathbf{R}$}

- Un minimum de 20 séances, trois fois par semaine, dont deux supervisées

- Des exercices de haute intensité donnent de meilleurs résultats

- Un entraînement avec intervalle peut être utile

- Une combinaison d'un entraînement en endurance ou en aérobie et d'un renforcement musculaire ou d'un travail en résistance

- Un entraînement des membres inférieurs et supérieurs

\section{Efficacité de la réhabilitation respiratoire}

$\mathrm{Au}$ cours des deux dernières décennies, l'efficacité de la RR a bien été démontrée par de larges études randomisées et contrôlées, et soutenue par des méta-analyses, telle par exemple la Cochrane Review publiée en 2006 par Lacasse ${ }^{[42]}$. Les bénéfices d'une RR multidisciplinaire concernent à la fois les patients, les dispensateurs des soins et aussi les autorités publiques (Fig. 4).

\section{Effets sur la qualité de vie}

Ainsi, la RR permet d'améliorer la qualité de vie des patients atteints de BPCO ${ }^{[43-49]}$. Si la mesure de la qualité de vie peut

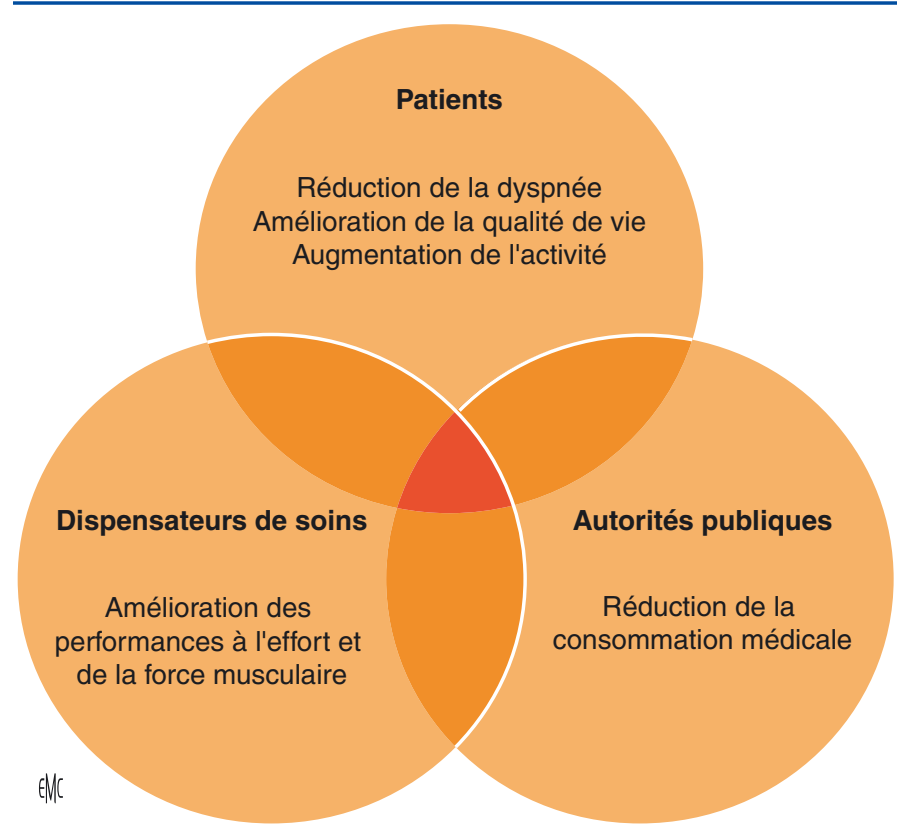

Figure 4. Bénéfices de la réhabilitation respiratoire.

se faire avec l'aide d'un questionnaire générique, ce sont principalement les questionnaires spécifiques validés dans la BPCO qui sont en pratique utilisés, tel le questionnaire respiratoire de St-George (SGRQ qui évalue trois domaines: les symptômes respiratoires, l'activité et l'impact psychosocial de la maladie) ${ }^{[50]}$ et le Chronic Respiratory Questionnaire (CRQ qui évalue quatre domaines: la dyspnée, la fatigue, l'affectivité, la maîtrise) [51]. Cependant, dans l'évaluation des effets de la RR, le CRQ semble un peu plus sensible que le SGRQ dans la détection d'une amélioration à court terme ${ }^{[52]}$. L'amélioration des scores du CRQ et du SGRQ suite à un programme de RR dépasse la différence minimale cliniquement significative : dix points pour le CRQ et quatre points pour le SGRQ ${ }^{[42]}$. De plus, un argument en faveur d'une utilisation plus systématique de la RR est l'amélioration de la qualité de vie par la RR qui est du même ordre, voire supérieure, aux bénéfices obtenus par les traitements médicamenteux ${ }^{[53]}$.

\section{Effets sur la dyspnée}

La dyspnée, qui est un facteur limitant important de l'effort et une source d'anxiété, est améliorée par un programme de RR [38, 42-46, 53-56]. Parmi les mécanismes impliqués dans cette " désensibilisation " de la dyspnée à l'effort, nous relevons une diminution des besoins ventilatoires pour une charge donnée ${ }^{[57]}$ et une réduction de l'hyperinflation dynamique [58].

\section{Effets sur la tolérance à l'effort}

Un programme de RR augmente les capacités physiques à l'effort telles que la puissance maximale atteinte $[42,43,55,57,58]$, la consommation maximale en $\mathrm{O}_{2}{ }^{[42,55]}$, le déplacement vers la droite du seuil ventilatoire et l'amélioration du test de marche de 6 minutes [43-46, 49], la force des muscles locomoteurs et l'endurance sur bicyclette ergométrique ou tapis roulant $[43$, 55]. Lacasse et al., dans leur méta-analyse, ont pu montrer que l'effet d'un programme de RR sur la charge maximale tolérée, mesurée lors d'une épreuve d'effort, est de l'ordre de 8,4 W [42], un résultat significatif mais finalement plutôt modeste car la charge maximale du patient BPCO est le plus souvent limitée par son système ventilatoire. Étant donné que la fonction pulmonaire et donc la ventilation maximale ne sont pas améliorées par le réentraînement à l'effort, l'effet d'un programme de RR sur la capacité maximale fonctionnelle ne peut être très important. En revanche, l'effet de la RR sur un effort submaximal tel que le test de la marche de 6 minutes, test moins dépendant de la capacité maximale ventilatoire, est 
beaucoup plus évident. Cette même méta-analyse [42] a ainsi montré que la distance parcourue lors d'un test de la marche de 6 minutes augmente en moyenne de $48 \mathrm{~m}$, amélioration qui s'approche des $54 \mathrm{~m}^{[59]}$ généralement considérés comme la différence minimale cliniquement significative (minimally important difference [MID]). Mais chez les patients BPCO, une telle valeur pourrait sous-estimer les résultats de la RR. À partir d'une autre méta-analyse [60], il a été suggéré dans la BPCO une valeur seuil de 35 mètres et plus récemment, Holland et al. [61] ont déterminé le MID à 25 mètres. Enfin, l'amélioration du périmètre de marche au test de la marche de 6 minutes dépend du nombre de sessions que compte le programme de RR: plus de 28 sessions permettent une augmentation de $50,3 \mathrm{~m}$, tandis que moins de 28 sessions mènent à une progression de seulement 34,5 m ${ }^{[53]}$. De plus, les programmes dépassant les 6 mois sont supérieurs aux programmes d'une plus courte durée, et ceux bien supervisés entraînent une amélioration du test de la marche de 6 minutes supérieure aux programmes où la supervision est moins intensive [62]. De plus, en maximisant la capacité respiratoire par la bronchodilatation, on peut aussi améliorer les résultats sur la tolérance à l'effort après un programme de RR [63].

Une autre approche pour améliorer la capacité respiratoire maximale est l'administration durant l'effort de mélange gazeux de plus faible densité que l'air. Ainsi, respirer un mélange gazeux où l'azote est remplacé par de l'hélium (héliox) réduit le travail respiratoire en raison probablement d'une diminution des turbulences et de l'hyperinflation dynamique. Une revue récente de la littérature a permis de conclure que par rapport à l'air ambiant, l'héliox améliorait la capacité à l'effort et dans une moindre mesure la dyspnée à l'effort des patients BPCO [64] Cependant, en plus des difficultés inhérentes à son utilisation, son coût élevé limite considérablement son application en routine clinique. Enfin, si la plupart des études suggèrent que les effets cliniques de la RR tendent à s'estomper graduellement avec le temps, ces derniers peuvent néanmoins perdurer au moins jusqu'à 2 ans de la fin d'un programme de 6 mois [43, 46]. Il est donc essentiel de poursuivre l'entraînement au domicile (marche quotidienne et/ou vélo d'appartement pendant $30 \mathrm{~min})$, chez un kinésithérapeute ou dans un centre sportif, voire de participer à un programme de maintenance dans le centre de RR, pour conserver au maximum les acquis.

\section{Effets sur l'activité physique}

Les patients BPCO présentent un niveau d'activité physique moindre que les sujets sains, et cela n'est d'ailleurs pas sans conséquences. Ainsi, Garcia-Aymerich et al. [65] ont mis en évidence, dans une étude prospective de cohorte où l'activité physique était estimée par questionnaire, que les patients BPCO avec un niveau d'activité physique bas et très bas, présentaient un risque d'hospitalisation et de mortalité supérieur à ceux qui rapportaient un niveau d'activité modéré ou élevé. Par ailleurs, il serait légitime de penser que l'amélioration de la tolérance à l'effort, obtenue par la RR, va se traduire par une augmentation de l'activité physique chez ces patients dans leur vie quotidienne. Cependant, cette amélioration après un programme de RR n'est pas toujours garantie [66]. Plusieurs équipes mènent actuellement des travaux sur ce sujet, notamment depuis le développement de nouveaux outils sophistiqués (BodyMedia ${ }^{\circledR}$ SenseWear ${ }^{\circledR}$ Armband) qui permettent de quantifier l'activité physique. Récemment, une équipe a pu mettre en évidence que le temps passé à marcher par des patients BPCO dans la vie quotidienne n'était pas significativement amélioré après 3 mois de RR multidisciplinaire mais bien après 6 mois ${ }^{[67]}$. Ceci est corrélé à d'autres études qui ont démontré que de véritables changements de comportement ne s'obtiennent chez les patients qu'après 6 mois.

\section{Effets sur les muscles respiratoires}

Si le renforcement systématique des muscles inspiratoires chez les patients BPCO n'est pas considéré comme un composant essentiel en routine de la RR [30], une revue de littérature a montré que lorsque la charge de travail était appropriée
(> $30 \%$ à $40 \%$ de la pression inspiratoire maximale et contrôlée), l'entraînement des muscles inspiratoires donnait une réduction de la dyspnée au repos et à l'effort, une amélioration de l'endurance et de la force des muscles inspiratoires, et une tendance à l'augmentation, cependant non significative, de la capacité à l'effort [68]. Ceci peut justifier l'entraînement spécifique des muscles inspiratoires chez les patients ayant une dysfonction des muscles inspiratoires ${ }^{[30]}$. Cependant, un entraînement global peut aussi par lui-même améliorer la force des muscles inspiratoires [43, 69].

\section{Effets sur les muscles des membres supérieurs}

L'entraînement des muscles des membres supérieurs peut soulager les symptômes liés à leur utilisation dans la vie courante [70]. Il doit faire partie intégrante d'un programme de RR car il améliore la capacité d'effort des membres supérieurs et réduit la ventilation et le coût en consommation maximale d'oxygène $\left(\mathrm{VO}_{2}\right)$ durant une activité des membres supérieurs [30].

\section{Effets sur la dépression et l'anxiété}

La RR réduit la morbidité psychosociale générale (dépression, anxiété, hostilité, tensions, etc.) [47, 48, 71, 72], même en l'absence d'un soutien psychologique spécifique durant le programme de RR [47]. Il va de soi que l'amélioration de la capacité à l'effort et de l'autonomie du patient, la réduction de la dyspnée et de l'isolement social jouent un rôle important dans la réduction de la morbidité psychologique du patient BPCO.

\section{Effets sur la consommation médicale et les exacerbations}

Les patients BPCO représentent un lourd fardeau pour les deniers publics des états: consultations, examens techniques, hospitalisations, traitements, invalidités, etc. ${ }^{[73]}$ et en particulier lors d'exacerbations entraînant une hospitalisation. Parmi les objectifs rencontrés par la $\mathrm{RR}$, il y a aussi la réduction du nombre de ces exacerbations [45], d'admissions et de jours d'hospitalisation pour exacerbation l'année suivant la RR [74-76]. Dans une étude récente chez des patients venant d'être hospitalisés pour exacerbation, il a été observé, 3 mois après leur sortie de l'hôpital, une réduction significative du taux d'exacerbations nécessitant une nouvelle hospitalisation chez les patients suivant un programme de RR ambulatoire de 8 semaines par rapport à ceux qui n'en recevaient pas (7\% versus $33 \%)$ [77]. La RR permet ainsi de réduire le recours aux soins de santé et le coût financier de la prise en charge globale du patient BPCO $[75,78]$. La RR en externe semble avoir un meilleur rapport coût/efficacité que les programmes nécessitant une hospitalisation ${ }^{[79] .}$

\section{Effets sur la fonction respiratoire et déclin annuel du VEMS}

La RR améliorant les composantes systémiques de la BPCO, il n'est donc pas étonnant que le VEMS au repos ne soit guère modifié $[43,46,49,53,54,56]$. Cependant, les études rapportées jusqu'à ce jour concernaient des programmes de relativement courtes durées. Une étude contrôlée récente évaluant l'efficacité d'un programme ambulatoire d'une durée de 3 ans [80] a objectivé, dans le groupe revalidé, une réduction significative du déclin du VEMS sur les 3 ans (74 $\mathrm{ml}$ versus $149 \mathrm{ml}$ pour le groupe contrôle). Cette étude est la première qui suggère qu'une RR prolongée peut ralentir le déclin de la fonction respiratoire du patient BPCO. Si ces résultats devaient être confirmés par d'autres études, nous aurions non seulement une nouvelle justification pour entreprendre chez nos patients BPCO une RR, mais aussi des programmes de plus longues durées.

\section{Effets sur le poids}

Une intervention nutritionnelle combinée avec un réentraînement bien supervisé peut entraîner une augmentation du 
poids corporel et de la masse musculaire chez des patients BPCO dénutris [81-83], mais ce gain peut être limité en raison de la prise de corticostéroïdes par voie systémique [81]. Une étude a montré, chez 85 patients BPCO et admis dans un programme de RR de 7 semaines, que par rapport au placebo, un supplément nutritif à base d'hydrates de carbone améliorait de manière significative le poids et, dans le sous-groupe de patients ayant un IMC supérieur à $19 \mathrm{~kg} / \mathrm{m}^{2}$, la capacité à l'effort [83]. De plus, une étude a montré que l'injection intramusculaire de stéroïdes anabolisants (nandrolone décanoate) pourrait encore renforcer les effets favorables de l'intervention nutritionnelle chez des patients BPCO revalidés tels que l'augmentation de la masse maigre et le gain sur la force des muscles respiratoires [82]. Les effets positifs des androgènes ne sont toutefois pas retrouvés en l'absence d'un programme de RR ${ }^{[84]}$. Cependant, même si les anabolisants augmentent la masse maigre des patients BPCO, leur utilisation en routine lors d'un programme de RR dans la BPCO n'est toutefois pas recommandée [30].

\section{Effets sur la survie}

Aucune des grandes études randomisées et contrôlées n'a pu démontrer une amélioration de la survie dans le groupe suivant un programme de $\mathrm{RR}$, en le comparant à un groupe traité de façon conventionnelle [43, 46, 54, 75, 85]. Ce manque d'évidence n'indique pas nécessairement que la RR n'exerce aucun effet sur la survie. En effet, ces études n'ont pas été conçues pour détecter une différence de survie. Par exemple, dans l'étude de Ries et al. ${ }^{[54]}$, la survie à 6 ans dans le groupe revalidé (programme ambulatoire de 8 semaines) était de $67 \%$ contre $56 \%$ pour le groupe contrôle (différence non significative). Regroupant les résultats de plusieurs études, Troosters et al. ${ }^{[53]}$ ont montré que la RR permet de réduire de $31 \%$ sur 18 mois le risque relatif de décès par rapport au groupe non revalidé, une différence toutefois non statistiquement significative. Pourtant, la possibilité de démontrer un effet positif de la RR sur la survie est bien réelle, car elle agit favorablement sur des déterminants importants de la survie, tels que l'IMC, la force musculaire, la masse maigre et la tolérance à l'effort, et enfin le nombre d'exacerbations [24, 25, 86-90]. Ainsi, si la survie des patients BPCO est largement déterminée par la simple mesure du VEMS [91], d'autres éléments de cette maladie systémique ont aussi une valeur prédictive en termes de survie [24, 25, 92, 93]. Ces paramètres ont été inclus dans l'index BODE (B pour body mass index, VEMS en pourcentage de sa valeur théorique comme indicateur d'obstruction des voies aériennes [O], score à l'échelle de dyspnée modifiée du Medical Research Council [D] et distance parcourue en 6 minutes comme indicateur de la capacité d'effort [E]), dont on a démontré qu'il peut prédire la survie avec une précision supérieure au VEMS seul ${ }^{[94]}$. Alors si la RR ne peut améliorer le VEMS, elle peut en revanche améliorer l'index BODE d'une façon significative ${ }^{[49,}{ }^{74]}$. Ainsi, il n'est pas déraisonnable d'avancer l'hypothèse que la RR améliorerait la survie.

\section{6 \\ Point fort}

\section{Efficacité de la RR}

La RR a un effet bénéfique sur la tolérance à l'effort, les symptômes de dyspnée et la qualité de vie perçue par le patient; dans le même temps, il y a une réduction de la consommation médicale. L'effet sur la survie n'a pas été établi de manière irréfutable bien qu'une tendance favorable soit perceptible.

\section{Conclusion}

Ainsi les patients souffrant de BPCO, avec activité physique réduite ou ayant des symptômes non maîtrisés malgré un traitement médical bien conduit, peuvent et doivent bénéficier d'une RR en ambulatoire ou, si cela n'est pas possible, en hospitalisation. Les patients âgés ou souffrant d'une BPCO très sévère ne doivent pas être exclus des programmes de RR. La réhabilitation doit être multidisciplinaire et adaptée à chaque patient. Elle permet en effet d'améliorer la dyspnée, la capacité à l'effort et l'activité globale du patient, mais également la qualité de vie des patients au travers d'une plus grande autonomie dans les activités de la vie quotidienne et d'une diminution de leur état dépressif ou d'anxiété. De plus, elle permet de réduire le recours aux soins de santé et donc le coût. Aujourd'hui, les médecins ne peuvent donc plus ignorer l'intérêt de la RR dans la prise en charge des patients BPCO. Mais force est de constater que la RR reste encore hélas sous utilisée en Europe, alors qu'en Amérique du Nord, elle occupe déjà une place importante dans la prise en charge de patients BPCO.

\section{Références}

[1] Global Initiative for Chronic Obstructive Lung Disease (GOLD). Global strategy for the diagnosis, management, and prevention of chronic obstructive pulmonary disease-2006. Available at www.goldcopd.com. Accessed March 15, 2007.

[2] Celli BR, MacNee W, and committee members. Standards for the diagnosis and treatment of patients with COPD: a summary of the ATS/ERS position paper. Eur Respir J 2004;23:932-46.

[3] Corhay JL, Nguyen Dang D, Schees P, Salamun I, Bury T, Pirnay F, et al. La réhabilitation pulmonaire dans la bronchopneumopathie chronique obstructive. Rev Med Liege 2008;63:677-83.

[4] Maltais F, LeBlanc P, Simard C, Jobin J, Berube C, Bruneau J, et al. Skeletal muscle adaptation to endurance training in patients with chronic obstructive pulmonary disease. Am J Respir Crit Care Med 1996;154:442-7.

[5] Rutschmann OT, Janssens JP, Vermeulen B, Sarasin FP. Knowledge of guidelines for the management of COPD: a survey of primary care physicians. Respir Med 2004;98:932-7.

[6] Nici L, Donner C, Wouters E, Zuwallack R, Ambrosino N, Bourbeau J, et al. American Thoracic Society/European Respiratory Society. Am J Respir Crit Care Med 2006;173:1390-413.

[7] Debigaré R, Côté C, Malteus F. Peripheral muscle wasting in chronic obstructive pulmonary disease; clinical relevance and mechanisms. Am J Respir Crit Care Med 2001;164:1712-7.

[8] Jones N, Killian K. Limitation of exercise in chronic airway obstruction. In: Cherniacks NS, editor. Chronic obstructive pulmonary disease. Philadelphia: WB Saunders; 1991. p. 196-206.

[9] Killian KJ, Leblanc P, Martin DH, Summers E, Jones NL, Campbell EJ. Exercise capacity and ventilatory, circulatory, and symptom limitation in patients with airflow limitation. Am Rev Respir Dis 1992;146: 935-40.

[10] Rabinovich R, Bastos R, Ardite E. Mitochondrial dysfunction in COPD patients with low body mass index. Eur Respir J 2007;29:643-50.

[11] Gosker HR, Zeegers M, Wouters E, Schols AM. Muscle fibre type shifting in the vastus lateralis of patients with COPD is associated with disease severity: a systemic review and meta-analysis. Thorax 2007; 62:944-9.

[12] Engelen M, Schols M, Larnres R, Wouters E. Different patterns of chronic tissue wasting among patients with chronic obstructive pulmonary disease. Clin Nutr 1999;18:275-80.

[13] Whittom F, Jobin J, Simard P, Leblanc P, Simard C, Bernard S, et al. Histochemical and morphological characteristics of the vastus lateralis muscle in COPD patients. Comparison with normal subjects and effects of exercise training. Med Sci Sports Exerc 1998;30:1467-74.

[14] Levine S, Kaiser L, Leferovich J, Tikunov B. Cellular adaptation in the diaphragm in chronic obstructive pulmonary disease. $N$ Engl J Med 1997;337:1799-806.

[15] Gosker HR, Hesselink MK, Duimel H, Ward KA, Schols AM. Reduced mitochondrial density in the vastus lateralis muscle of patients with COPD. Eur Respir J 2007;30:73-9.

[16] Hill NS. Pulmonary rehabilitation. Proc Am Thor Soc 2006;3:66-74.

[17] Young P, Dewse M, Fergusson W, Kolbe J. Respiratory rehabilitation in chronic obstructive pulmonary disease: predictors of nonadherence. Eur Respir J 1999;13:855-9. 
[18] Katsura H, Kanemaru A, Yamada K, Motegi T, Wakabayashi R, Kida K. Long-term effectiveness of an inpatient pulmonary rehabilitation program for elderly COPD patients: comparison between youngelderly and old-elderly groups. Respirology 2004;9:230-6.

[19] Di Meo F, Pedone C, Lubich S, Pizzoli C, Traballesi M, Incalzi RA. Age does not hamper the response to pulmonary rehabilitation of COPD patients. Age Ageing 2008;37:530-5.

[20] Niederman MS, Clemente PH, Fein AM, Feinsilver SH, Robinson DA, Ilowite JS, et al. Benefits of a multidisciplinary rehabilitation program. Improvements are independant of lung function. Chest 1991;99: 798-804.

[21] Young P, Dewse M, Fergusson W, Kolbe J. Respiratory rehabilitation in chronic obstructive pulmonary disease: predictors of nonadherence. Eur Respir J 1999;13:855-9.

[22] Solway S, Brooks D, Lau L, Goldstein R. The short-term effect of a rollator on functional exercise capacity among individuals with severe COPD. Chest 2002;122:56-65.

[23] Probst VS, Troosters T, Coosemans I, Spruit MA, Pitta F, Decramer M, et al. Mechanisms of improvement in exercise capacity using a rollator in patients with COPD. Chest 2004;126:1102-7.

[24] Landbo C, Prescott E, Lange P, Vestbo J, Almdal TP. Prognostic value of nutritional status in chronic obstructive pulmonary disease. Am J Respir Crit Care Med 1999;160:1856-61.

[25] Schols AM, Slangen J, Volovics L, Wouters EF. Weight loss is a reversible factor in the prognosis of chronic obstructive pulmonary disease. Am J Respir Crit Care Med 1998;157:1791-7.

[26] Baarends EM, Schols AM, Mostert R, Wouters EF. Peak exercise response in relation to tissue depletion in patients with chronic obstructive pulmonary disease. Eur Respir J 1997;10:2807-13.

[27] Shoup R, Dalsky G, Warner S, Davies M, Connors M, Khan M, et al. Body composition and health-related quality of life in patients with obstructive airways disease. Eur Respir J 1997;10:1576-80.

[28] Weekens CE, Emery PW, Elia M. Dietary counselling and food fortification in stable COPD: a randomised trial. Thorax 2009;64:326-31.

[29] Zigmond AS, Snaith RP. The hospital anxiety and depression scale. Acta Psychiatr Scand 1983;67:361-70.

[30] Ries AL, Bauldoff GS, Carlin BW, Casaburi R, Emery CF, Mahler DA, et al. Pulmonary rehabilitation: Joint ACCP/AACVPR EvidenceBased Clinical Practice Guidelines. Chest 2007;131:4S-42S.

[31] Lemoigne F, Desplans J, Lonsdorfer E, Lonsdorfer J, Miffre C, Perruchini JM, et al. Stratégies de la réhabilitation respiratoire. Rev Mal Respir 2005;22:7S100-7S111.

[32] Ouksel H, Gautier V, Bajon D, Barel P, Veale D, Tardif C, et al. La réhabilitation respiratoire à domicile: données de la littérature, aspects pratiques et médico-économiques. Rev Mal Respir 2004;21:727-35.

[33] Maltais F, Bourbeau J, Shapiro S. Effects of home-based pulmonary rehabilitation in patients with chronic obstructive pulmonary disease. Ann Intern Med 2008;149:869-78.

[34] Beauchamp MK, Nonoyama M, Goldstein RS, Hill K, Dolmage TE, Mathur S, et al. Interval versus continuous training in individuals with chronic obstructive pulmonary disease- a systematic review. Thorax 2010;65:157-64.

[35] Puhan MA, Busching G, Schunemann HJ, vanOort E, Zaugg C, Frey M. Interval versus continuous high-intensity exercise in chronic obstructive pulmonary disease: a randomized trial. Ann Intern Med 2006; 145:816-25.

[36] Vogiatzis I, Nanas S, Roussos C. Interval training as an alternative modality to continuous exercise in patients with COPD. Eur Respir J 2000;20:12-9.

[37] Mahler DA, Ward J, Mejia-Alfaro R. Stability of dyspnea ratings after exercise training in patients with COPD. Med Sci Sports Exerc 2003; 35:1083-7.

[38] Sillen MJ, Janssen PP, Akkermans MA, Wouters EF, Spruit MA. The metabolic response during resistance training and electrical stimulation (NMES) in patients with COPD, a pilot study. Respir Med 2008;102: 786-9.

[39] Vivodtzev I, Pépin JL, Vottero G, Mayer V, Porsin B, Lévy P, et al. Improvement in quadriceps strength and dyspnea in daily tasks after 1 month of electrical stimulation in severely deconditionned and malnourished COPD. Chest 2006;129:1540-8.

[40] Bourbeau J, Julien M, Maltais F, Rouleau M, Beaupré A, Begin R, et al. Reduction of hospital utilization in patients with chronic obstructive pulmonary disease: a disease-specific self-management intervention. Arch Intern Med 2003;163:585-91.
[41] Gadoury MA, Schwartzman K, Rouleau M, Maltais F, Julien M, Beaupré A, et al. Self-management reduces both short- and long-term hospitalisation in COPD. Eur Respir J 2005;26:853-7.

[42] Lacasse Y, Goldstein R, Lasserson TJ, Martin S. Pulmonary rehabilitation for chronic obstructive pulmonary disease. Cochrane Database Syst Rev 2006(4):CD003793.

[43] Troosters T, Gosselink R, Decramer M. Short- and long-term effects of outpatient rehabilitation in patients with chronic obstructive pulmonary disease: a randomized trial. Am J Med 2000;109:207-12.

[44] Goldstein RS, Gort EH, Stubbing D, Avendano MA, Guyatt GH. Randomised controlled trial of respiratory rehabilitation. Lancet 1994 ; 344:1394-7.

[45] Wedzicha JA, Bestall JC, Garrod R, Garnham R, Paul EA, Jones PW. Randomized controlled trial of pulmonary rehabilitation in severe chronic obstructive pulmonary disease patients, stratified with the MRC dyspnoea scale. Eur Respir J 1998;12:363-9.

[46] Guell R, Casan P, Belda J, Sangenis M, Morante F, Guyatt GH, et al. Long-term effects of outpatient rehabilitation of COPD: a randomized trial. Chest 2000;117:976-83.

[47] Güell R, Resqueti V, Sangenis M, Morante F, Martorell B, Casan P, et al. Impact of pulmonary rehabilitation on psychosocial morbidity in patients with severe COPD. Chest 2006;129:899-904.

[48] Paz-Diaz H. Montes de OM, Lopez JM, Celli BR. Pulmonary rehabilitation improves depression, anxiety, dyspnea and health status in patients with COPD. Am J Phys Med Rehabil 2007;86:30-6.

[49] Shahin B, Germain M, Pastene G, Viallet N, Annat G. Outpatient pulmonary rehabilitation in patients with chronic obstructive pulmonary disease. Int J Chron Obstruct Pulmon Dis 2008;3:155-62.

[50] Jones PW, Quirk FH, Baveystock CM, Littlejohn P. A self-complete measure of health status for chronic airflow limitation. Am Rev Respir Dis 1992;145:1321-7.

[51] Guyatt GH, Berman LB, Townsend M, Pugsley SO, Chambers LW. A measure of quality of life for clinical trials in chronic lung disease. Thorax 1987; 42:773-8

[52] Singh SJ, Sodergren SC, Hyland ME, Williams J, Morgan MD. A comparison of three disease-specific and two generic health-status measures to evaluate the outcome of pulmonary rehabilitation in COPD. Respir Med 2001;95:71-7.

[53] Troosters T, Casaburi R, Gosselink R, Decramer M. State of the Art. Pulmonary rehabilitation in chronic obstructive pulmonary disease. Am J Respir Crit Care Med 2005;172:19-38.

[54] Ries AL, Kaplan RM, Limberg TM, Prewitt LM. Effects of pulmonary rehabilitation on physiologic and psychosocial outcomes in patients with chronic obstructive pulmonary disease. Ann Intern Med 1995;122: 823-32.

[55] Lacasse Y, Wong E, Guyatt GH, King D, Cook DJ, Goldstein RS. Metaanalysis of respiratory rehabilitation in chronic obstructive pulmonary disease. Lancet 1996;348:1115-9.

[56] O'Donnell DE, McGuire M, Samis L, Webb KA. The impact of exercise reconditioning on breathlessness in severe chronic airflow limitation. Am J Respir Crit Care Med 1995;152:2005-13.

[57] Casabruni R, Porszasz J, Burns MR, Carithers ER, Chang RS, Cooper CB. Physiologic benefits of exercice training in rehabilitation of patients with severe chronic obstructive pulmonary disease. Am J Respir Crit Care Med 1997;155:1541-51.

[58] Porszasz J, Emtner M, Goto S, Somfay A, Whipp BJ, Casabruni R. Exercice training decreases ventilatory requirements and exerciceinduced hyperinflation at submaximal intensities in patients with COPD. Chest 2005;128:2025-34.

[59] Redelmeier DA, Bayoumi AM, Goldstein R, Guyatt G. Interpreting small differences in functional status: the six minute walk test in chronic lung disease patients. Am J Respir Crit Care Med 1997;155:1278-82.

[60] Puhan MA, Mador MJ, Held U, Goldstein R, Guyatt GH, Schunemann HJ. Interpretation of treatment changes in 6-minute walk distance in patients with COPD. Eur Respir J 2008;32:637-43.

[61] Holland AE, Hill CJ, Rasekaba T, Lee A, Naughton MT, McDonald CF. Updating the minimal important difference for six-minute walk distance in patients with chronic obstructive pulmonary disease. Arch Phys Med Rehabil 2010;91:221-5.

[62] Lacasse Y, Brosseau S, Milne S. Pulmonary rehabilitation for COPD. Cochrane Database Syst Rev 2002(3):CD003793.

[63] Casaburi R, Kukafka D, Cooper CB, Witek Jr. TJ, Kesten S. Improvement in exercise tolerance with the combination of tiotropium and pulmonary rehabilitation in patients with COPD. Chest 2005;127: 809-17.

[64] Hunt t, Williams MT, Frith P, Schembri D. Heliox, dyspnoea and exercice in COPD. Eur Respir Rev 2010;19:30-8. 
[65] Garcia-Aymerich J, Lange P, Benet M, Schnohr P, Anto JM. Regular physical activity reduces hospital admission and mortality in chronic obstructive pulmonary disease: a population based cohort study. Thorax 2006;61:772-8.

[66] Troosters T, Gosselink R, Janssens W, Decramer M. Exercice training and pulmonary rehabilitation: new insights and remaining challenges. Eur Respir Rev 2010;19:24-9.

[67] Pitta F, Troosters T, Probst VS, Langer D, Decramer M. Gosselink. Are patients with COPD more active after pulmonary rehabilitation? Chest 2008; 134:273-80

[68] Lötters F, Van Tol B, Kwakkel G, Gosselink R. Effects of controlled inspiratory muscle training in patients with COPD: a meta-analysis. Eur Respir J 2002;20:570-6.

[69] O’Donnell DE, McGuire M, Samis L, Webb KA. General exercise training improves ventilatory and peripheral muscle strength and endurance in chronic airflow limitation. Am J Respir Crit Care Med 1998; 157:1489-97.

[70] Gigliotti F, Coli C, Bianchi R, Grazzini M, Stendardi L, Castellani C, et al. Arm exercise and hyperinflation in patients with COPD. Chest 2005; 128: $1225-32$

[71] Emery CF, Schein RL, Hauck ER, MacIntyre NR. Psychological and cognitive outcomes of a randomized trial of exercise among patients with chronic obstructive pulmonary disease. Health Psychol 1998;17: 232-40.

[72] de Godoy DV, de Godoy RF. A randomized controlled trial of the effect of psychotherapy on anxiety and depression in chronic obstructive pulmonary disease. Arch Phys Med Rehabil 2003;84:1154-7.

[73] Mapel DW, Hurley JS, Frost FJ, Petersen HV, Picchi MA, Coultas DB. Health care utilization in chronic obstructive pulmonary disease. A case-control study in a health maintenance organization. Arch Intern Med 2000;160:2653-8.

[74] Cote CG, Celli BR. Pulmonary rehabilitation and the BODE index in COPD. Eur Respir J 2005;26:630-6.

[75] Griffiths TL, Burr ML, Campbell IA, Lewis-Jenkins V, Mullins J, Shiels K, et al. Results at 1 year of outpatient multidisciplinary pulmonary rehabilitation: a randomised controlled trial. Lancet 2000; 355:362-8.

[76] Hudson LD, Tyler ML, Petty TL. Hospitalization needs during an outpatient rehabilitation program for severe chronic airway obstruction. Chest 1976;70:606-10.

[77] Seymour MJ, Moore L, Jolley CL, Ward K, Creasey J, Steier JS, et al. Outpatient pulmonary rehabilitation following acute exacerbations of COPD. Thorax 2010;65:423-8.

[78] Griffiths TL, Phillips CJ, Davies S, Burr ML, Campbell IA. Cost effectiveness of an outpatient multidisciplinary pulmonary rehabilitation programme. Thorax 2001;56:779-84.

[79] Goldstein RS, Gort EH, Guyatt GH, Feeny D. Economic analysis of respiratory rehabilitation. Chest 1997;112:370-9.

[80] Stav D, Raz M, Shpirer I. Three years of pulmonary rehabilitation: inhibit the decline in airflow obstruction, improves exercise endurance time, and body-mass index, in chronic obstructive pulmonary disease. BMC Pulmon Med 2009;9:26-30.

[81] Creutzberg EC, Wouters EF, Mostert R, Weling-Scheepers CA, Schols AM. Efficacy of nutritional supplementation therapy in depleted patients with chronic obstructive pulmonary disease. Nutrition 2003; 19:120-7.
[82] Schols AM, Soeters PB, Mostert R, Pluymers RJ, Wouters EF. Physiologic effects of nutritional support and anabolic steroids in patients with chronic obstructive pulmonary disease. A placebocontrolled randomized trial. Am J Respir Crit Care Med 1995;152: 1268-74.

[83] Steiner MC, Barton RL, Singh SJ, Morgan DL. Nutritional enhancement of exercise performance in chronic obstructive pulmonary disease: a randomized controlled trial. Thorax 2003;58: 745-51.

[84] Sharma S, Arneja A, McLean L, Duerksen D, Leslie W, Sciberras D, et al. Anabolic steroids in COPD: a review and preliminary results of a randomized trial. Chron Respir Dis 2008;5:169-76.

[85] Engstrom CP, Persson LO, Larsson S, Sullivan M. Long-term effects of a pulmonary rehabilitation programme in outpatients with chronic obstructive pulmonary disease: a randomized controlled study. Scand J Rehabil Med 1999;31:207-13.

[86] Gray-Donald K, Gibbons L, Shapiro SH, MacKlem PT, Martin JG. Nutritional status and mortality in chronic obstructive pulmonary disease. Am J Respir Crit Care Med 1996;153:961-6.

[87] Martinez FJ, Foster G, Curtis JL, Criner G, Weinmann G, Fishman A, et al. Predictors of mortality in patients with emphysema and severe airflow obstruction. Am J Respir Crit Care Med 2006;173:1326-34.

[88] Soler-Cataluna JJ, Martinez-Garcia MA, Roman Sanchez P, Salcedo E, Navarro M, Ochando R. Severe acute exacerbations and mortality in patients with chronic obstructive pulmonary disease. Thorax 2005;60: 925-31.

[89] Marquis K, Debigare R, Lacasse Y, LeBlanc P, Jobin J, Carrier G, et al. Midthigh muscle cross-sectional area is a better predictor of mortality than body mass index in patients with chronic obstructive pulmonary disease. Am J Respir Crit Care Med 2002;166:809-13.

[90] Vestbo J, Prescott E, Almdal T, Dahl M, Nordestgaard BG, Andersen T, et al. Body mass, fat-free body mass, and prognosis in patients with chronic obstructive pulmonary disease from a random population sample. Am J Respir Crit Care Med 2006;173:79-83.

[91] Anthonisen NR, Wright EC, Hodgkin JE. Prognosis in chronic obstructive pulmonary disease. Am Rev Respir Dis 1986;133:14-20.

[92] Gerardi DA, Lovett L, Benoit-Connors ML, Reardon JZ, ZuWallack RL. Variables related to increased mortality following outpatient pulmonary rehabilitation. Eur Respir J 1996;9:431-5.

[93] Nishimura K, Izumi T, Tsukino M, Oga T. Dyspnea is a better predictor of 5-year survival than airway obstruction in patients with COPD. Chest 2002;121:1434-40.

[94] Celli BR, Cote CG, Marin JM, Casanova C, Montes de Oca M, Mendez RA, et al. The body-mass index, airflow obstruction, dyspnea, and exercise capacity index in chronic obstructive pulmonary disease. N Engl J Med 2004;350:1005-12.

\section{Pour en savoir plus}

Préfaut C, Ninot G. La réhabilitation du malade respiratoire chronique. Paris: Elsevier Masson; 2009 (502p).

Groupe Alvéole SPLF. La réhabilitation respiratoire. Guide pratique. Paris: Imothep MS; 2008 (252p).

J.-L. Corhay, Professeur de clinique, chef de clinique (jlcorhay@chu.ulg.ac.be).

D. Dang Nguyen, Chef de clinique.

T. Bury, Professeur.

F. Pirnay, Professeur, directeur du Centre de réhabilitation pulmonaire.

R. Louis, Professeur, chef de service.

Service de pneumologie et de réhabilitation respiratoire, Centre hospitalier universitaire Sart-Tilman B35 4000 Liège, Belgique.

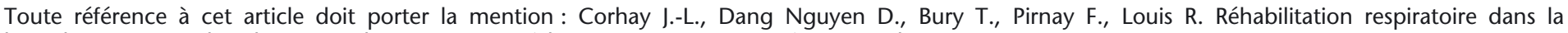
bronchopneumopathie chronique obstructive. EMC (Elsevier Masson SAS, Paris), Pneumologie, 6-040-L-65, 2011.

Disponibles sur www.em-consulte.com

Arbres décisionnels
Iconographies supplémentaires
Vidéos / Animations
Documents légaux
Information au patient
Informations supplémentaires
Auto-

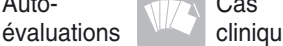

